\title{
For the M. Hargittai issue
}

\author{
Roald Hoffmann ${ }^{1}$
}

Received: 12 June 2015/Accepted: 12 June 2015/Published online: 21 July 2015

(C) Springer Science+Business Media New York 2015

For chemistry there is little better than a reliable structure. It delimits speculation-is that $\mathrm{AY}_{3}$ molecule planar with threefold symmetry, or is it $\mathrm{T}$ or $\mathrm{Y}$ shaped? A good structure does not stop there. It begins a conversation, for with it in hand we can begin to think of reaction mechanisms - what amino acid could swing into place to deliver a proton to a molecule bound just this way in an active site of an enzyme?

Magdi Hargittai has for 40 years provided us with reliable structures of fleeting, metastable molecules. These are so much harder to subject to trustworthy structural analysis than molecules peacefully resident in a crystal. And she has consistently given us the one thing more valuable than a reliable structure. This is the understanding of the "why"-why a molecule is nonlinear, when a Jahn-Teller distortion will be static and when dynamic, the workings of the Renner effect, and of large amplitude vibrations. For calculation itself is far from understanding. But the computer's rain of numbers can be shaped into comprehension in the mind (and words, and pictures, and equations!) of an intelligent scientist, one attuned to both chemistry and physics, one sensitive to how what happens in one molecule is connected to all molecules. Magdi Hargittai is such a scientist par excellence, who determines good structures and strives for accompanying understanding. It is not easily done, yet in every paper she writes, one can feel this pull to the "why."

It is a pleasure to join her friends in the tribute to her care and wisdom that this issue represents.
Roald Hoffmann

rh34@cornell.edu

1 Department of Chemistry and Chemical Biology, Cornell University, Baker Laboratory, Ithaca, NY 14853-1301, USA 